\title{
On acoustic propagation in three-dimensional rectangular ducts with flexible walls and porous linings*
}

\author{
by \\ Jane B Lawrie \\ Email: Jane.Lawrie@brunel.ac.uk \\ Department of Mathematics, Brunel University, Uxbridge, UB8 3PH, UK
}

\begin{abstract}
The focus of this paper is towards the development of hybrid analytic-numerical modematching methods for model problems involving three-dimensional ducts of rectangular cross-section and with flexible walls. Such methods require firstly closed form analytic expressions for the natural fluid-structure coupled waveforms that propagate in each duct section and secondly the corresponding orthogonality relations. It is demonstrated how recent theory [Lawrie, Proc. R. Soc. A. 465, 2347-2367 (2009)] may be extended to a wide class of three-dimensional ducts, for example, those with flexible walls and a porous lining (modelled as an equivalent fluid) or those with a flexible internal structure such as a membrane (the "drum-like" silencer). Two equivalent expressions for the eigenmodes of a given duct can be formulated. For the ducts considered herein, the first ansatz is dependent on the eigenvalues/eigenfunctions appropriate for wave propagation in the corresponding two-dimensional duct with flexible walls, whilst the second takes the form of a Fourier series. The latter has several advantages: in particular, no "rootfinding" is involved and the method is appropriate for ducts in which the flexible wall is orthotropic. The first ansatz, however, provides important information about the orthogonality properties of the eigenmodes.
\end{abstract}

\section{Introduction}

Systems of ducts or pipes are incorporated into the design of many buildings, aircraft and other engineering structures. The purpose of such ducts varies: circular cylindrical geometries have application, for example, to car exhaust systems, wave propagation in co-axial cables and noise emission by aeroengines $[1,2,3,4,5,6,7,8]$ whereas ducts of rectangular cross-section are more usual in heating ventilation and air-conditioning (HVAC) systems $[9,10,11,12,13,14]$. Whatever the application there is a need to

\footnotetext{
*Portions of this work were presented in "Acoustic propagation in 3-D, rectangular ducts with flexible walls", Proceedings of the Joint 159th Meeting of the Acoustical Society of America and NOISE-CON 2010, Baltimore, U.S.A., April 2010[12].
} 
understand scattering of the guided waves by non-uniformities or discontinuities. Depending on the exact circumstances such scattering may be viewed as either a positive or a negative attribute of the system. For example, on the one hand this phenomenon underpins the design of a many classes of silencer yet on the other hand simple discontinuities between, say, strips of lining can seriously limit the performance of such a device [6]. A range of analytic and numerical methods are available for the solution of model problems involving the scattering of waves in ducts. Each approach has it own strengths and limitations and so the choice of method will depend not only on the duct geometry but also upon the aims of the investigation.

For geometries comprising semi-infinite duct sections the Wiener-Hopf technique [15] can prove to be a powerful tool. The method is most appropriate for the solution of 2-D (or 3-D) boundary value problems involving a governing equation together with a two-part boundary condition imposed along one infinite coordinate line $[16,4]$ (for example, one condition for $x<0, y=0$ and a different condition for $x>0, y=0)$. Several extensions to the method are available [15]; the two most noteworthy being the modified Wiener-Hopf technique which enables the approach to be applied to boundary value problems involving three-part boundary data, and matrix formulations $[5,8,17]$ which often arise when the mixed conditions are imposed for $x<0$ and $x>0$ but at different values of $y$. It should be remarked that whilst the Wiener-Hopf technique can be used to study ducting systems with or without breakout, it cannot easily be applied to boundary value problems describing closed ducts featuring a height change.

The Galerkin procedure offers another effective approach. This method has been successfully employed in the study of the "drum-like" silencer (which comprises an expansion chamber together with a membrane positioned such that the fluid within the chamber is separated from the main body of fluid $[18,11])$. The fluid velocity on the membrane is expressed in terms of the basis functions (in this case, Fourier sine modes) and the sound field within the duct is represented by an infinite sum of Fourier integrals, each forced by a velocity distribution that is zero on the rigid sections of wall and equal to one of the basis functions on the flexible section. The modal amplitudes are then determined by enforcing the equation of motion for the membrane. In the case of the drum-like silencer this approach offers the advantage that no "root-finding" is necessary, however, some physical quantities such as the phase speeds of the natural duct modes are not readily deduced.

Mode-matching (MM) methods are a popular approach to investigating ducting systems in which breakout can be neglected. Numerical MM [20] is highly versatile but does not always permit insight into the underlying physical properties. Hybrid analyticnumerical schemes offer an interesting balance in which the analysis is taken as far as 
possible in order to formulate a robust system for numerical solution. The advantage of such an approach is two-fold: it enables physical insight into the underlying scattering processes and the final numerical computations may be less time intensive. For 2-D model problems analytic-numerical MM methods are well established [19, 21, 22, 23, 24] and can be applied to ducts with a wide range of realistic wall conditions (rigid, soft, flexible etc). Such methods proceed by expressing the fluid velocity potential in each duct region as an expansion in terms of the natural duct modes. The relevant orthogonality relations are then invoked in order to impose the appropriate continuity conditions at the interface between duct sections. The problem is thus reduced to that of solving an infinite system of linear equations the subject of which is usually the modal coefficients. In contrast to the 2-D case, most 3-D analytic MM approaches are suitable only for ducts in which the cross-sections are either circular or rectangular and then only for simple wall conditions (soft, hard, impedance). Two interesting examples have recently appeared in the literature: azimuthal non-uniformity is notoriously difficult to address and thus the hybrid analytical-numerical scheme offered by $\mathrm{Bi}$ et al [7] is of particular interest; the modelling of poroelastic foam within a silencer is equally challenging and Nennig et al. [3] have developed a scheme which takes into consideration the compressional and shear waves within the foam.

This article is concerned with wave propagation in 3-D ducts of rectangular crosssection that would appropriately form part of an HVAC system. In such systems sound from fans and/or motors can propagate for significant distances via reflections from the internal walls or as vibration along the wall itself. Traditional analytic methods for modelling sound propagation in such ducts have tended to neglect the latter, primarily because the exact form of the propagating waves was unknown. Recent research [25], however, has established both the analytic waveforms corresponding to acoustic propagation in a 3-D duct with flexible walls and also many of their mathematical properties. This advance prepares the ground for the development of full hybrid analytic-numerical MM solutions to model problems directly related to noise control issues in HVAC systems.

The current article offers several extensions to the theory presented by Lawrie [25]. Its applicability to a wide class of 3-D ducts is demonstrated, including configurations with both flexible walls and an internal layer of porous material and those in which one boundary is both flexible and orthotropic. Three different ducts are considered: the unlined duct with flexible walls; the lined duct again with flexible walls and a rigid walled duct the interior of which is partitioned by a membrane (this latter duct comprising the reactive component in the drum-like silencer [18, 11]). For each duct a closed form exact expression for the fluid-structure coupled travelling waveforms is presented. These 
are validated by comparison of the phase speeds or attenuation curves with existing graphs in the literature (all of which are produced by alternative means such as the Rayleigh-Ritz method or finite elements).

The article is organised as follows. In Sec. 2 the analytic forms for the acoustic waves that propagate in an unlined 3-D duct with one elastic wall are revisited. The ansatz presented herein is more general than that of Lawrie [25] enabling both clamped and simply supported corner conditions to be considered. The next section deals with the extension of the theory to the case of acoustic propagation in a 3-D duct with one elastic wall and a porous lining (which is modelled as an equivalent fluid with complex density and propagation coefficient). Section 4 focuses on the 3-D drum-like silencer. The analysis of Sec. 2 is first applied to the case in which the membrane is isotropic. It is then explained why this approach fails should the membrane be orthotropic; an alternative method for deriving the eigenfunctions is demonstrated. It is found that varying the tensions in the two principle directions has a significant effect on the phase speeds of the propagating modes. Finally, the application of the theory presented in Sec. 2-4 to the development of hybrid analytic-numerical MM schemes for the solution of model problems involving 3-D ducts of this class is discussed in Sec. 5.

\section{Propagation in an unlined 3-D duct}

The first duct configuration to be considered comprises a 3-D duct of rectangular crosssection occupying the region $-\infty<\bar{x}<\infty, 0 \leq \bar{y} \leq \bar{a},-\bar{b} \leq \bar{z} \leq \bar{b}$ where $(\bar{x}, \bar{y}, \bar{z})$ are dimensional Cartesian coordinates. A compressible fluid, of density $\rho_{a}$ and sound speed $c_{a}$, occupies the interior region of the duct, whilst the region exterior to the duct is in vacuo. Harmonic time dependence, $e^{-i \omega \bar{t}}$, is assumed and the full velocity potential is expressed in terms of the time independent potential by $\bar{\Phi}(\bar{x}, \bar{y}, \bar{z}, \bar{t})=\bar{\phi}(\bar{x}, \bar{y}, \bar{z}) e^{-i \omega \bar{t}}$. It is convenient to non-dimensionalise the boundary value problem with respect to length and time scales $k^{-1}$ and $\omega^{-1}$ respectively, where $\omega=c_{a} k$ and $k$ is the fluid wavenumber. Thus, non-dimensional co-ordinates are defined by $x=k \bar{x}$ etc. Similarly, $\bar{\phi}=\omega \phi / k^{2}$ etc. This non-dimensionalisation is used throughout the article.

The duct comprises three rigid walls, lying along $y=0,-b \leq z \leq b,-\infty<x<\infty$ and $z= \pm b, 0 \leq y \leq a,-\infty<x<\infty$ and is closed by a thin elastic plate lying along $y=a,-b \leq z \leq b,-\infty<x<\infty$ (see figure 1). Due to the coupling between the fluid and plate motions, the travelling waves are not separable in form. Without loss of generality, however, it can be assumed that they propagate in the positive $x$ direction. 

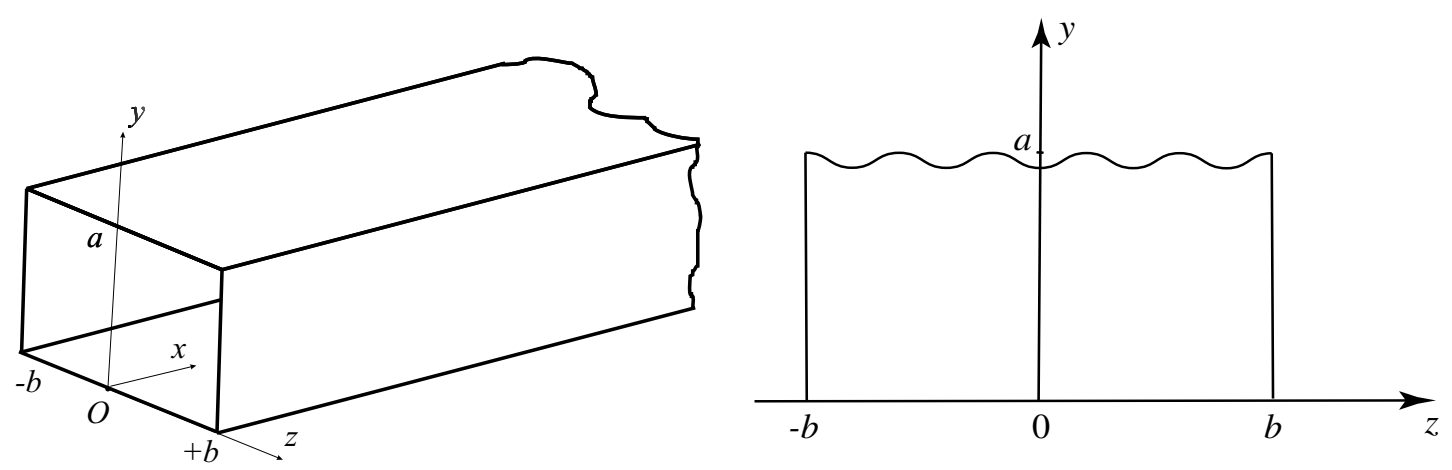

Figure 1: The unlined 3-D duct and its yz cross-section.

The non-dimensional, time-independent velocity potential then assumes the form

$$
\phi(x, y, z)=\sum_{n=0}^{\infty} B_{n} \psi_{n}(y, z) e^{i s_{n} x}, \quad x>0
$$

where $B_{n}$ is the amplitude of the $\mathrm{n}^{\text {th }}$ travelling wave, $s_{n}$ is the axial wavenumber (assumed to be either positive real or have positive imaginary part) and the nonseparable eigenmodes $\psi_{n}(y, z), n=0,1,2, \ldots$ are to be determined.

It is convenient, initially, to treat the wavenumber as a continuous variable $s$ rather than a discrete set of values, $s_{n}$. Then, $\psi_{n}(y, z)=\psi\left(s_{n}, y, z\right)$ and the potential $\psi(s, y, z)$ satisfies reduced wave equation:

$$
\left\{\frac{\partial^{2}}{\partial y^{2}}+\frac{\partial^{2}}{\partial z^{2}}+\chi^{2}-s^{2}\right\} \psi(s, y, z)=0
$$

where $0 \leq y<a,-b \leq z \leq b$ and the non-dimensional fluid wavenumber is $\chi=1$. The normal component of fluid velocity vanishes at the three rigid walls which, implies:

$$
\begin{array}{ll}
\frac{\partial \psi}{\partial y}=0, & y=0, \quad|z| \leq b, \\
\frac{\partial \psi}{\partial z}=0, & z= \pm b, \quad 0 \leq y \leq a .
\end{array}
$$

The boundary condition that describes the deflections of the thin elastic plate bounding the top of the duct is

$$
\left\{\left(\frac{\partial^{2}}{\partial z^{2}}-s^{2}\right)^{2}-\mu^{4}\right\} \psi_{y}-\alpha \psi=0, y=a,|z| \leq b
$$

where $\mu$ is the in vacuo plate wavenumber and $\alpha$ a fluid-loading parameter. These quantities are defined in (10) below. Recollect that the elastic plate meets the rigid duct walls along the edges $y=a, z= \pm b,-\infty<x<\infty$. Thus, in addition, to the 
governing equation and boundary conditions outlined above, it is necessary to apply "corner conditions" along these edges to describe how the plate is connected to the rigid side wall. Two options will be considered: clamped and pin-jointed corner conditions.

It is useful to discuss the form of $\psi(s, y, z)$ in relation to (3) and (4). Following [25], it is assumed that symmetric eigenmodes (that is, those that satisfy $\psi(s, y,-z)=$ $\psi(s, y, z))$ have the form:

$$
\psi(s, y, z)=\sum_{m=0}^{\infty} E_{m}(s) Y_{m}(y) \cosh \left(\tau_{m}(s) z\right)
$$

where $E_{m}(s)$ and $\tau_{m}(s), m=0,1,2, \ldots$ depend on the parameter $s$, whilst $Y_{m}(y)$, $m=0,1,2, \ldots$ are the eigenfunctions appropriate for wave propagation in the 2-D duct corresponding to the $x y$ cross-section of the 3 -D waveguide (see figure 2). Thus, it is known a priori that $Y_{m}^{\prime}(0)=0$ where the prime denotes differentiation with respect to the argument, $y$. In order to impose the rigid wall condition at $z= \pm b, 0 \leq y \leq a$, it is necessary that

$$
\sum_{m=0}^{\infty} E_{m}(s) \tau_{m}(s) \sinh \left(\tau_{m}(s) b\right) Y_{m}(y)=0 .
$$

It is immediately apparent that the eigenfunctions $Y_{m}(y), m=0,1,2, \ldots$ must be linearly dependent. Note that the structure of antisymmetric modes (for which $\psi^{a}(s, y,-z)=$ $\left.-\psi^{a}(s, y, z)\right)$ is obtained from (6) simply by replacing $\cosh \left(\tau_{m}(s) z\right)$ with $\sinh \left(\tau_{m}(s) z\right)$.

\subsection{The underlying 2-D eigensystem}

In view of the importance of the eigenfunctions $Y_{n}(y)$, it is appropriate to review wave propagation in the underlying 2-D system. The relevant 2-D duct lies in the region $0 \leq y \leq a,-\infty<x<\infty$. The upper boundary comprises an elastic plate whilst the base, lying along $y=0$, is rigid. The velocity potential satisfies the 2-D Helmholtz's equation with unit non-dimensional wavenumber and the elastic plate is described by the 2-D plate equation (that is (5) but with $\frac{\partial}{\partial z}=0$ ).

Disturbances comprising fluid-structural waves propagating in the positive $x$-direction may be expressed in the form:

$$
\phi(x, y)=\sum_{n=0}^{\infty} A_{n} Y_{n}(y) e^{i \zeta_{n} x}, \quad x>0
$$

where $Y_{n}(y)=\cosh \left(\gamma_{n} y\right), A_{n}$ is the modal amplitude, $\zeta_{n}=\sqrt{\gamma_{n}^{2}+1}$ and is defined to be positive real or have positive imaginary part. On substituting $Y(y)=\cosh (\gamma y)$ where $\gamma=\left(\zeta^{2}-1\right)^{1 / 2}, \gamma(0)=-i$, into the plate equation, it is found that the eigenvalues $\gamma_{n}$, 


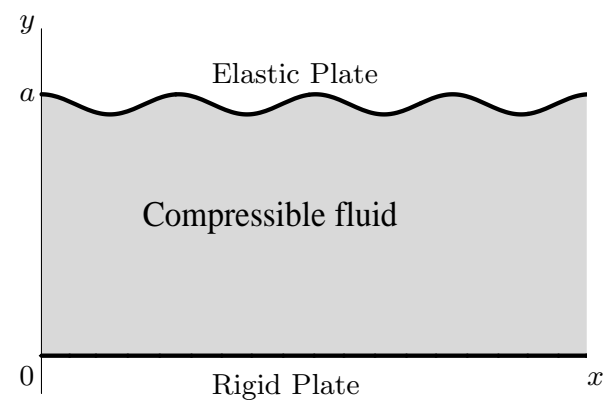

Figure 2: The $x y$ cross-section of the unlined 3-D duct.

$n=0,1,2, \ldots$ are the roots of $K(\gamma)=0$ where

$$
K(\gamma)=\left\{\left(\gamma^{2}+1\right)^{2}-\mu^{4}\right\} Y^{\prime}(a)-\alpha Y(a) .
$$

Here $\mu$ and $\alpha$ are the in vacuo plate wavenumber and fluid-loading parameter mentioned above. They are defined by

$$
\mu^{4}=\frac{12\left(1-\nu^{2}\right) c_{a}^{2} \rho_{p}}{k^{2} \bar{h}^{2} E} ; \quad \alpha=\frac{12\left(1-\nu^{2}\right) c_{a}^{2} \rho_{a}}{k^{3} \bar{h}^{3} E}
$$

where $E$ is Young's modulus, $\rho_{p}$ is the density of the plate, $\bar{h}$ is the dimensional plate thickness, $\rho_{a}$ is the density of the compressible fluid and $\nu$ is Poisson's ratio. The roots of $K(\gamma)=0$ have the following properties: i) they occur in pairs, $\pm \gamma_{n}$; ii) there is a finite number of real roots; iii) there is an infinite number of imaginary roots; iv) complex roots, $\pm \gamma_{c}$ and $\pm \gamma_{c}^{*}$ occur for some frequency ranges. It is assumed that no root is repeated.

For real and imaginary roots, the convention is adopted that the $+\gamma_{n}$ roots are either positive real or have positive imaginary part. They are ordered sequentially, real roots first and then by increasing imaginary part. Thus, $\gamma_{0}$ is always the largest real root. As indicated in iv) above, should a complex root, say $\gamma_{c}$, lie in the upper half of the complex $\gamma$-plane, then minus the complex conjugate, $-\gamma_{c}^{*}$, will also lie in this half plane. Such pairs are incorporated into the sequence of roots according to the magnitude of their imaginary part, and in the order $\gamma_{c}$ followed by $-\gamma_{c}^{*}$. (Since complex roots arise as the imaginary plate mode approaches a "cut-off" duct mode, a guide to the values of $k$ for which these are to be found can be obtained by solving $i \mu=\sqrt{1-\frac{n^{2} \pi^{2}}{k^{2} \bar{a}^{2}}}, n=1,2,3 \ldots$ where $\mu$ is given in (10) and $\bar{a}$ is the dimensional height of the duct.)

The eigenfunctions $Y_{m}(y), m=0,1,2, \ldots$ satisfy the generalised orthogonality relation (OR):

$$
\alpha \int_{0}^{a} Y_{m}(y) Y_{j}(y) d y=C_{j} \delta_{j m}-\left(\gamma_{m}^{2}+\gamma_{j}^{2}+2\right) Y_{j}^{\prime}(a) Y_{m}^{\prime}(a)
$$


where $\delta_{j m}$ is the Kronecker delta and $C_{m}$ is given by

$$
C_{m}=\left.\frac{Y_{m}^{\prime}(a)}{2 \gamma_{m}} \frac{d}{d \gamma} K(\gamma)\right|_{\gamma=\gamma_{m}}
$$

The eigenfunctions $Y_{j}(y), j=0,1,2, \ldots$ are linearly dependent for $0 \leq y \leq a$ :

$$
\sum_{n=0}^{\infty} \frac{Y_{n}^{\prime}(a) Y_{n}(y)}{C_{n}}=\sum_{n=0}^{\infty} \frac{\gamma_{n}^{2} Y_{n}^{\prime}(a) Y_{n}(y)}{C_{n}}=0
$$

and satisfy the identities:

$$
\sum_{n=0}^{\infty} \frac{\left[Y_{n}^{\prime}(a)\right]^{2}}{C_{n}}=0, \quad \sum_{n=0}^{\infty} \frac{\gamma_{n}^{2}\left[Y_{n}^{\prime}(a)\right]^{2}}{C_{n}}=1
$$

In addition, a Green's function can be constructed:

$$
\begin{aligned}
\alpha \sum_{n=0}^{\infty} \frac{Y_{n}(v) Y_{n}(y)}{C_{n}} & =\delta(y-v)+\delta(y+v) \\
& +\delta(y+v-2 a), \quad 0 \leq v, y \leq a
\end{aligned}
$$

where $\delta(y)$ is the Dirac delta function. This expression is crucial to proving that the eigenfunction expansion representation of a suitable smooth function, say $f(y)$ does indeed converge point-wise to that function. Results (11)-(15) are established in reference $[26]$.

\subsection{Eigenmodes for the $3-\mathrm{D}$ case}

From (6), and bearing in mind (13), the exact, closed form expression for the symmetric eigenfunctions, may be expressed as

$$
\psi(s, y, z)=\sum_{m=0}^{\infty} \frac{\left\{1-Q(s) \gamma_{m}^{2}\right\} Y_{m}^{\prime}(a) Y_{m}(y) \cosh \left(\tau_{m}(s) z\right)}{C_{m} \tau_{m}(s) \sinh \left(\tau_{m}(s) b\right)}
$$

where $\gamma_{m}^{2}+\tau_{m}^{2}(s)-s^{2}+1=0$. (The anti-symmetric modes are obtained from (16) on replacing $\cosh \left(\tau_{m}(s) z\right)$ with $\sinh \left(\tau_{m}(s) z\right)$ and $\sinh \left(\tau_{m}(s) b\right)$ with $\cosh \left(\tau_{m}(s) b\right)$.) It is clear that (16) satisfies the rigid wall conditions at $y=0, z=0$ and the elastic plate equation at $y=a$. The rigid wall conditions at $z= \pm b$ reduce to

$$
\sum_{m=0}^{\infty} \frac{\left\{1-Q(s) \gamma_{m}^{2}\right\} Y_{m}^{\prime}(a) Y_{m}(y)}{C_{m}}=0
$$

and the reader may verify by comparison with (13) that this is indeed zero. Thus, there remains two corner conditions to apply and it is these that dictate the form of the unknown function $Q(s)$ and the values of admissible wavenumbers, $s_{n}, n=0,1,2, \ldots$. 


\subsubsection{Clamped corner conditions}

For the case in which the plate is clamped along the edges $y=a, z= \pm b,-\infty<x<\infty$ the appropriate corner conditions are

$$
\frac{\partial \psi}{\partial y}=\frac{\partial^{2} \psi}{\partial y \partial z}=0, \quad y=a, \quad z= \pm b .
$$

In view of the second identity in (14) and the second condition of (18) it is clear that $Q(s)=0$. Thus, for clamped corners, the symmetric eigenfunctions have the form

$$
\psi(s, y, z)=\sum_{m=0}^{\infty} \frac{Y_{m}^{\prime}(a) Y_{m}(y) \cosh \left(\tau_{m}(s) z\right)}{C_{m} \tau_{m}(s) \sinh \left(\tau_{m}(s) b\right)} .
$$

On applying the first condition of (18) it is found that the admissible wavenumbers, $s_{n}$, for symmetric modes are given by

$$
L(s)=\sum_{m=0}^{\infty} \frac{\left[Y_{m}^{\prime}(a)\right]^{2} \cosh \left\{\tau_{m}(s) b\right\}}{C_{m} \tau_{m}(s) \sinh \left\{\tau_{m}(s) b\right\}}=0
$$

where $\tau_{m}(s)=\left(s^{2}-\gamma_{m}^{2}-1\right)^{1 / 2}$. (The antisymmetric wavenumbers are deduced by interchanging the sinh and cosh in (20)). The reader is reminded that, having determined the form of the eigenfunctions and the admissible wavenumbers, the eigenmodes are given by $\psi_{n}(y, z)=\psi\left(s_{n}, y, z\right)$.

Being a sum over the eigenvalues for the underlying 2-D system, the characteristic function, $L(s)$, is of a somewhat unusual form. Further, it exhibits an infinite number of asymptotes. The roots have the following properties: i) they occur in pairs, $\pm s_{n}$; ii) there is a finite number of real roots; iii) there is an infinite number of imaginary roots; iv) there is an infinite number of roots with non-zero real and imaginary parts. In order that (1) represents only waves that travel in the positive $x$ direction and/or decay exponentially as $x \rightarrow \infty$, the convention is adopted that the $+s_{n}$ roots are either positive real or have positive imaginary part. They are ordered sequentially, as described for $\gamma_{n}$, $n=0,1,2, \ldots$ in Sec. 2.1. This approach to ordering the eigenvalues/roots is henceforth adopted as standard.

The pertinent features of $L(s)$ and its roots are illustrated in figures 3 and 4 . In order that a useful comparison can be made, the physical parameters are the same as those chosen by Martin et al.[10]. Thus, the dimensional duct height and half width are $\bar{a}=0.09 \mathrm{~m}$ and $\bar{b}=0.053 \mathrm{~m}$. The upper surface comprises an aluminium plate of thickness $\bar{h}=0.0006 \mathrm{~m}$ and density $\rho_{=} 2700$. Young's modulus and Poisson's ratio are given by $E=7.2 \times 10^{10} \mathrm{Nm}^{-2}$ and $\nu=0.34$; whilst $c_{a}$ and $\rho_{a}$ are taken to be $344 \mathrm{~ms}^{-1}$ and $1.2 \mathrm{~kg} \mathrm{~m}^{-3}$ respectively. 

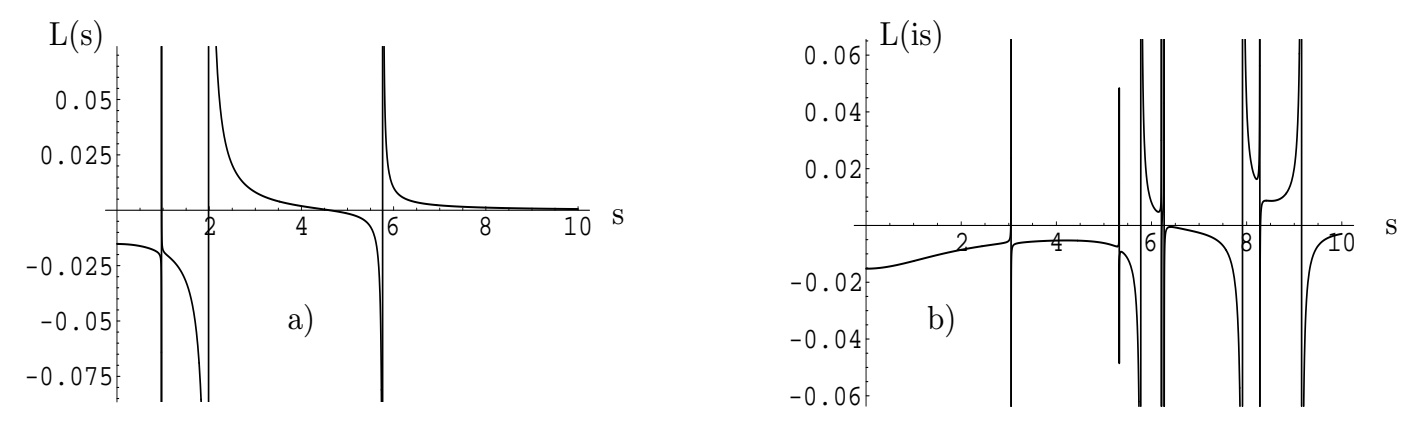

Figure 3: The characteristic function for the unlined 3-D duct (symmetric modes, clamped corners) plotted for a) real and b) imaginary arguments at $600 \mathrm{~Hz}$.

Figure 3 shows $L(s)$ (as given by (20)) plotted for real and imaginary arguments. The asymptotes are apparent and it can also be seen that roots often lie very close to an asymptote. The rapid change of gradient in the close proximity of a root can present problems for root finding algorithms such as the Newton-Raphson method. This situation is improved, however, by seeking the roots of $M(s)=0$ as opposed to $L(s)=0$ where

$$
M(s)=L(s)\left\{\sum_{m=0}^{\infty} \frac{1}{C_{m} \tau_{m}(s) \sinh \left\{\tau_{m}(s) b\right\}}\right\}^{-1} .
$$
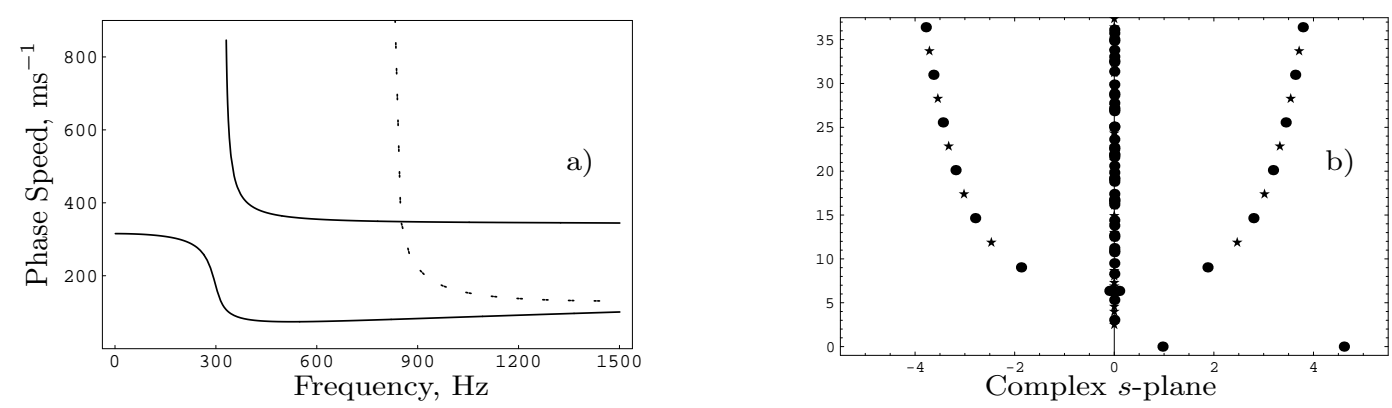

Figure 4: Clamped corners: a) phase speeds of the unattenuated symmetric (solid) and anti-symmetric (dashed) waves plotted against frequency; b) location of the symmetric (circles) and antisymmetric (stars) roots in the complex s-plane at 600Hz.

Figure 4(a) shows the phase speeds for the unattenuated symmetric and anti-symmetric modes for the frequency range $0-1500 \mathrm{~Hz}$. The dashed line is that for the first antisymmetric mode (which "cuts-on" at approximately $825 \mathrm{~Hz}$ ). The two solid curves are the phase speeds for the fundamental and first "higher-order" symmetric modes. The latter are in excellent agreement with those presented by Martin et al.[10], who deter- 
mined the phase speeds by constructing an approximate characteristic equation. They were, in fact, able to construct and compare two different approximations: one obtained via a variational approach in which the sound pressure was assumed constant and the plate displacement sinusoidal; the second again assumed constant pressure but utilised a more refined expression for the plate displacement. Both expressions were valid only for frequencies below the second symmetric cut-on. In contrast, the characteristic function, $L(s)$, (and its anti-symmetric counterpart) is exact and is valid for all frequencies - provided sufficient eigenvalues for the underlying 2-D case are known (an issue which can be avoided as will be discussed later). Figure 4(b), shows the location in the complex s-plane of both the symmetric and anti-symmetric roots at a frequency of $600 \mathrm{~Hz}$. There are two real roots (both of which correspond to symmetric modes), an infinite number of imaginary roots (corresponding to both symmetric and anti-symmetric modes) and also an infinite family of complex roots.

As mentioned above, the anti-symmetric modes are easily deduced on interchanging the hyperbolic functions in the symmetric eigenfunctions and in the characteristic equation. For this reason attention is, henceforth, restricted to symmetric modes defined for $0 \leq z \leq b$.

\subsubsection{Pin-jointed corner conditions}

For the case in which the plate is pin-jointed (simply supported) along the edges $y=a$, $z= \pm b,-\infty<x<\infty$ the appropriate corner conditions are

$$
\frac{\partial \psi}{\partial y}=\frac{\partial^{3} \psi}{\partial y \partial z^{2}}=0, \quad y=a, \quad z=b
$$

In this case the second condition of (22) yields

$$
Q(s)=\frac{\sum_{m=0}^{\infty} C_{m}^{-1}\left[Y_{m}^{\prime}(a)\right]^{2} \tau_{m}(s) \operatorname{coth}\left\{\tau_{m}(s) b\right\}}{\sum_{m=0}^{\infty} C_{m}^{-1} \gamma_{m}^{2}\left[Y_{m}^{\prime}(a)\right]^{2} \tau_{m}(s) \operatorname{coth}\left\{\tau_{m}(s) b\right\}}
$$

and, on applying the first condition of (22), it is found that the admissible wavenumbers, $s_{n}$, for the symmetric modes are given by $L(s)=0$ where

$$
L(s)=\sum_{m=0}^{\infty} \frac{\left\{1-Q(s) \gamma_{m}^{2}\right\}\left[Y_{m}^{\prime}(a)\right]^{2} \cosh \left\{\tau_{m}(s) b\right\}}{C_{m} \tau_{m}(s) \sinh \left\{\tau_{m}(s) b\right\}} .
$$

The characteristic function and its roots have properties similar to those for the clamped corner case. It is interesting to note, however, that the family of complex roots shown in figure 4(b) do not appear to be a feature for this set of corner conditions - although isolated complex roots do occasionally occur. Figure 5 shows the phase speeds of the symmetric eigenmodes and the location of the roots corresponding to this set of corner 
conditions at $600 \mathrm{~Hz}$. Again, for the purposes of comparison, the parameters of Martin et al.[10] are used. It is clear that the second and third symmetric modes cut-on at lower frequencies than for the clamped-corner case.
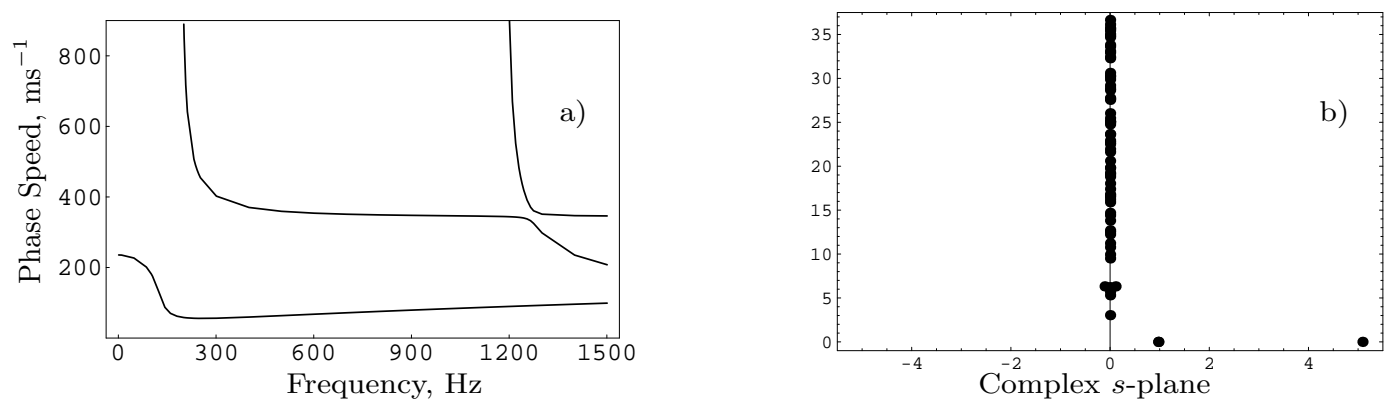

Figure 5: Pin-jointed corners: a) phase speeds of the unattenuated waves plotted against frequency; b) location of the roots in the complex s-plane at $600 \mathrm{~Hz}$.

\section{Propagation in a lined 3-D duct}

In this section the situation whereby a porous lining is inserted into the unlined 3-D duct described above is considered. It is worthwhile emphasising that the ansatz (6) is equally appropriate for symmetric eigenfunctions corresponding to acoustic propagation in a lined duct as it is for the unlined case: all that is necessary is to replace the 2-D eigenmode $Y_{n}(y), n=0,1,2, \ldots$

It is assumed that the lining occupies the region $d \leq y \leq a, 0 \leq z \leq b$, where $0<d<a$, then the $x y$ cross-section of the duct is shown in figure 6(a). The porous material is modelled as an equivalent fluid with complex density, $\rho_{\ell}$, and propagation coefficient, $\Gamma$. Thus, the non-dimensional wavenumber $\chi$ of equation (2) is now given by

$$
\chi=\left\{\begin{array}{cc}
1, & 0 \leq y<d \\
\Gamma, & d<y \leq a
\end{array} .\right.
$$

The complex propagation coefficient and density are evaluated using the established empirical formulae:

$$
\Gamma=1+i a_{1} \xi^{a_{2}}+a_{3} \xi^{a_{4}} ; \beta=\Gamma\left(1+a_{5} \xi^{a_{6}}+i a_{7} \xi^{a_{8}}\right)
$$

where $\beta=\rho_{\ell} / \rho_{a}, \xi=\rho_{a} f / \sigma$ in which $f$ is the frequency and $\sigma$ is the flow resistivity. The constants $a_{1}-a_{8}$ are determined experimentally and the typical values for various fibrous materials are well known[27, 28]. In this article the Delany and Bazley constants will be 
used, namely: $a_{1}=0.1890, a_{2}=-0.5950, a_{3}=0.0978, a_{4}=-0.7, a_{5}=0.0571, a_{6}=$ $-0.7540, a_{7}=0.0870, a_{8}=-0.7320$. It should be noted that $\Gamma$ and $\beta$ as defined above differ slightly from the equivalent quantities used in articles in which the chosen time dependence is $e^{i \omega t}$ (for example, Kirby[2]). For $e^{i \omega t}$ time dependence the complex propagation constant and density are related to those used herein by $\Gamma^{+}=(-i \Gamma)^{*}$ and $\beta^{+}=\beta^{*}$ where $^{*}$ denotes the complex conjugate.
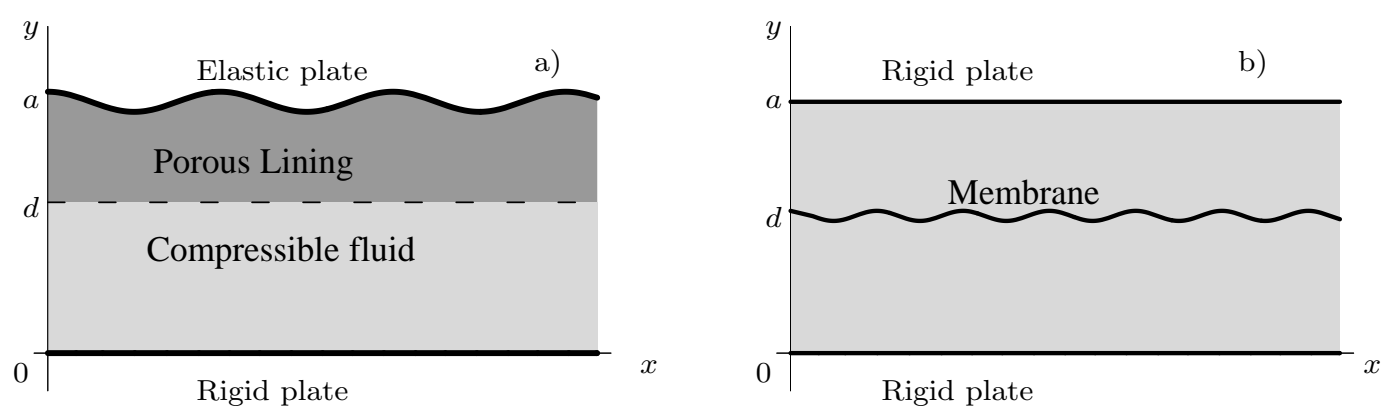

Figure 6: The $x y$ cross-sections of a) the lined 3-D duct (Sec. 3); b) the drum-like silencer (Sec. 4).

The 2-D velocity potential representing wave propagation in the positive $x$-direction is given by (8) in which $\zeta_{n}=\left(\gamma_{n}^{2}+1\right)^{1 / 2}=\left(\lambda_{n}^{2}+\Gamma^{2}\right)^{1 / 2}$ and the 2-D eigenfunctions are given by:

$$
Y_{n}(y)= \begin{cases}Y_{1 n}(y), & 0 \leq y<d \\ Y_{2 n}(y), & d<y \leq a\end{cases}
$$

where $Y_{1 n}(y)=\cosh \left(\gamma_{n} y\right)$ and

$$
\begin{aligned}
Y_{2 n}(y) & =\gamma_{n} \sinh \left(\gamma_{n} d\right) \frac{\sinh \left(\lambda_{n}(y-d)\right)}{\lambda_{n}} \\
& +\frac{1}{\beta} \cosh \left(\gamma_{n} d\right) \cosh \left(\lambda_{n}(y-d)\right) .
\end{aligned}
$$

Note that, $Y_{1 n}(d)=\beta Y_{2 n}(d)$ and $Y_{1 n}^{\prime}(d)=Y_{2 n}^{\prime}(d)$ which ensures continuity of pressure and normal velocity at the fluid/porous material interface. Also, $Y_{1 m}^{\prime}(0)=0$ which is consistent with the rigid base at $y=0$. The eigenvalues, $\gamma_{n}$ are the roots of $K(\gamma)=0$ where $K(\gamma)$ is given by

$$
K(\gamma)=\left\{\left(\gamma^{2}+1\right)^{2}-\mu^{4}\right\} Y_{2}^{\prime}(a)-\alpha Y_{2}(a) .
$$

where $Y_{2}(y)$ is obtained from (27) simply by replacing the discrete values $\gamma_{n}$ and $\lambda_{n}$, $n=0,1,2, \ldots$ with the similarly related continuous variables $\gamma$ and $\lambda=\left(\gamma^{2}+1-\Gamma^{2}\right)^{1 / 2}$. The plate wavenumber $\mu$ and fluid-loading parameter $\alpha$ are given by (10), however, in the definition of $\alpha, \rho_{a}$ is now replace by $\rho_{\ell}$. 
The eigenfunctions satisfy the generalised OR:

$$
\begin{aligned}
\alpha \int_{0}^{d} Y_{1 m} Y_{1 j} d y & +\alpha \beta \int_{d}^{a} Y_{2 m} Y_{2 j} d y \\
=\beta C_{j} \delta_{j m} & -\beta\left(\gamma_{m}^{2}+\gamma_{j}^{2}+2\right) Y_{2 j}^{\prime}(a) Y_{2 m}^{\prime}(a)
\end{aligned}
$$

where $C_{j}$ is given by (12). The eigenfunctions (26)-(27) also satisfy the identities (13) and (14). These can be verified numerically and, although not formally proven for this specific eigenproblem, are well established for a range of similar systems [26, 29].

On assuming clamped corners, the correct symmetric eigenfunctions and characteristic equation for the lined 3-D duct with one flexible wall are now given by equations (19) and (20) in conjunction with (26)-(27) and (12). Note that, in order for (2) to be satisfied $\gamma_{m}^{2}+\tau_{m}^{2}(s)+1-s^{2}=0$ and $\lambda_{m}^{2}+\tau_{m}^{2}(s)+\Gamma^{2}-s^{2}=0$.
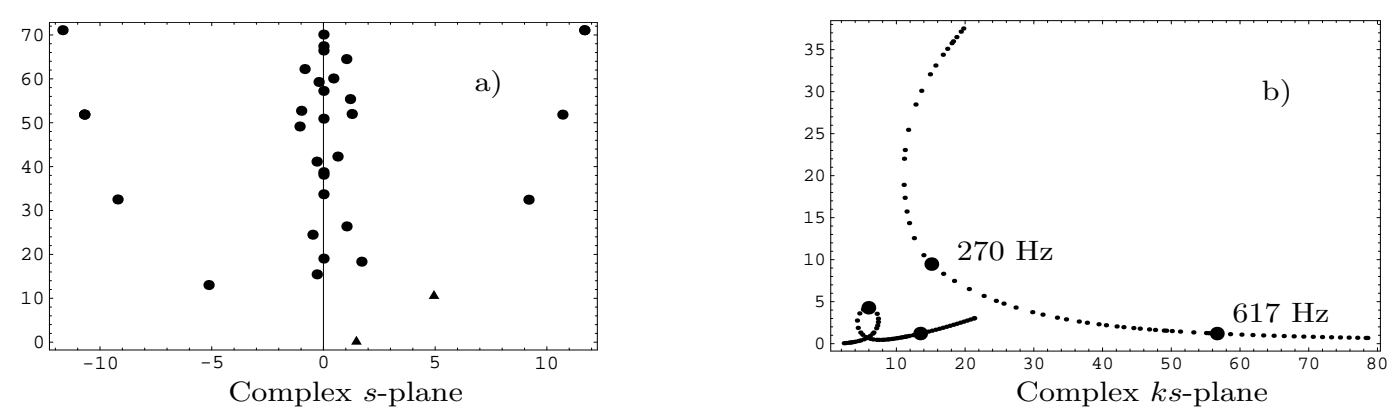

Figure 7: Lined duct: a) location of the non-dimensional roots in the complex $s$-plane at $170 \mathrm{~Hz}$ (the two least attenuated roots marked by triangles); b) path in the complex plane along which the dimensional least attenuated roots move as frequency increases (the larger points indicate the frequencies $270 \mathrm{~Hz}$ and $617 \mathrm{~Hz}$ respectively).

For this 3-D duct configuration the characteristic equation has no real roots and it is the attenuation of those modes with the smallest imaginary parts that is of interest. In order that a useful comparison can be made, the physical parameters used herein are those chosen by Astley et al.[9] Other than the plate thickness, these are the same as those chosen by Martin et al.[10], and are listed in Sec. 2.2. The plate thickness used here is $\bar{h}=0.00054 \mathrm{~m}$ whilst $\bar{d}=0.06 \mathrm{~m}$ and $\sigma=20000$ SI rayls $\mathrm{m}^{-1}$.

Figure $7(\mathrm{a})$ shows the location, in the complex plane, of the roots of characteristic equation, at a frequency of $170 \mathrm{~Hz}$. The two least attenuated modes are denoted by triangles. Figure 7(b) shows the loci in the complex plane of the two least attenuated dimensional wavenumbers, that is, $k s_{n}, n=0,1$. The paths are traversed from left to right with increasing frequency and the location of the wavenumbers at $270 \mathrm{~Hz}$ and $617 \mathrm{~Hz}$ are indicated. Figure 8 shows the axial attenuation, that is $20 k \Im\left(s_{n}\right) / \ln (10)$, of the two 


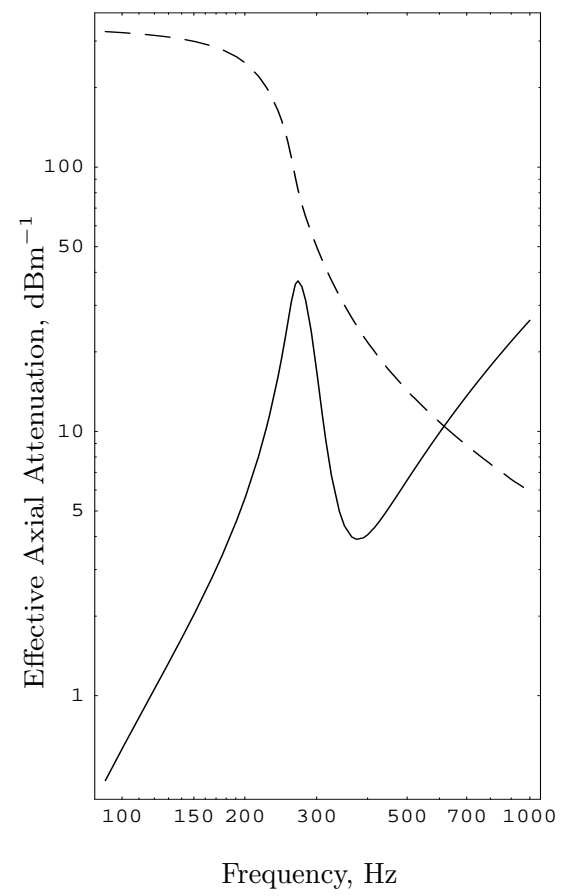

Figure 8: The axial attenuation (logarithmic scale) for the two least attenuated roots (dashed curve corresponds to the upper triangle in figure 7a)).

least attenuated modes $n=0,1$. Note that the maximum attenuation of the $s_{0}$ mode occurs at $270 \mathrm{~Hz}$ and that the attenuations of the two modes are equal at approximately $616 \mathrm{~Hz}$. The solid curve shows good agreement with its counterpart (reference [9]: figure 12) obtained by Astley et al. using a finite element scheme.

\section{Propagation in the drum-like silencer}

The final 3-D duct configuration to be considered is that which forms the reactive component for the drum-like silencer[18, 11]. This duct comprises a $3-\mathrm{D}$ rigid walled duct with an internal membrane lying along $y=d, 0 \leq z \leq b$ where $0<d<a$. The interior regions are filled with a compressible fluid of sound speed $c_{a}$ and density $\rho_{a}$. This duct configuration differs from the previous two in that not all the travelling waveforms are non-separable[19]. For ducts in which $p d-a q=0$ where $p$ and $q$ are integers, there are two families of eigenfunctions: those for which the fluid and membrane motions are coupled and those for which the motions are uncoupled. The latter are a subset of the usual rigid duct modes, that is, those that have zero velocity normal to the membrane 
at $y=d$ where $d=a q / p$. Thus, the fluid velocity potential is given by

$$
\begin{aligned}
\phi(x, y, z) & =\sum_{n=0}^{\infty} \sum_{n=0}^{\infty} A_{m n} U_{m n}(y, z) e^{i \eta_{m n} x} \\
& +\sum_{n=0}^{\infty} B_{n} \psi_{n}(y, z) e^{i s_{n} x}, \quad x>0
\end{aligned}
$$

where $U_{m n}(y, z)=\cos \left(\frac{m p \pi y}{a}\right) \cos \left(\frac{n \pi z}{b}\right)$ and $\eta_{m n}=\left(1-m^{2} \pi^{2} / a^{2}-n^{2} p^{2} \pi^{2} / b^{2}\right)^{1 / 2}$. As before, the potential $\psi(s, y, z)$ satisfies $(2)$ (with $\chi=1$ ) together with (3)-(4). In addition the upper surface of the duct is rigid and a membrane lies along $y=d, 0<d<a$, within the duct.

\subsection{Isotropic membrane}

For an isotropic membrane (in which the tensions in the $x$ and $z$ directions are the same) the appropriate boundary condition is:

$$
\left\{\frac{\partial^{2}}{\partial z^{2}}-s^{2}+\mu^{2}\right\} \psi_{y}-\alpha[\psi]_{d^{-}}^{d^{+}}=0, \quad 0 \leq z \leq b
$$

where $\mu=c_{a} / c_{m}$ and $\alpha=c_{a}^{2} \rho_{a} /(k T)$ in which $c_{m}=\left(T / \rho_{m}\right)^{1 / 2}$ is the in-vacuo speed of waves on the membrane, $T$ is the membrane tension and $\rho_{m}$ is the mass per unit area of the membrane. For such membranes, the 3-D coupled eigenmodes can be constructed using the method demonstrated in Sec. 2 and 3. It is again emphasised that the ansatz (6) is still appropriate for symmetric eigenmodes in this duct - all that is necessary is to replace $Y_{n}(y), n=0,1,2, \ldots$

The $x y$ cross-section of the 3 -D duct is shown in figure $6(\mathrm{~b})$. The velocity potential representing coupled wave propagation in the positive $x$-direction of this 2 -D configuration is given by (8) in which $\zeta_{n}=\left(\gamma_{n}^{2}+1\right)^{1 / 2}$ and the eigenfunctions are defined piece-wise (c.f. (26)). For this duct $Y_{1 n}(y)=\cosh \left(\gamma_{n} y\right)$ and

$$
Y_{2 n}(y)=\frac{\sinh \left(\gamma_{n} d\right)}{\sinh \left[\gamma_{n}(d-a)\right]} \cosh \left[\gamma_{n}(a-y)\right]
$$

Note that, $Y_{1 n}^{\prime}(d)=Y_{2 n}^{\prime}(d)$ which ensures continuity of normal velocity across the membrane and also that $Y_{1 n}^{\prime}(0)=Y_{2 n}^{\prime}(a)=0$. The eigenvalues, $\gamma_{n}, n=0,1,2, \ldots$, are the roots of the characteristic equation $K(\gamma)=0$ where

$$
K(\gamma)=\left(\gamma^{2}+1-\mu^{2}\right) \gamma \sinh (\gamma d)-\frac{\alpha \sinh (\gamma a)}{\sinh [\gamma(a-d)]}
$$

The functions $Y_{n}(y)$ satisfy the generalised OR[19]:

$$
\alpha \int_{0}^{a} Y_{n}(y) Y_{m}(y) d y+Y_{n}^{\prime}(d) Y_{m}^{\prime}(d)=C_{n} \delta_{m n}
$$


where $C_{n}$ is given by

$$
\left.C_{n}=\frac{Y_{n}^{\prime}(d)}{2 \gamma_{n}} \frac{d}{d \gamma} K(\gamma)\right\rfloor_{\gamma=\gamma_{n}} .
$$

(A similar OR has recently been presented for the case of a submerged elastic plate[30].) Again, the eigenfunctions, $Y_{n}(y)$ are linearly dependent but in this case, due to the lower order of the membrane equation, there is only one relation that expresses this[26]:

$$
\sum_{n=0}^{\infty} \frac{Y_{n}^{\prime}(d) Y_{n}(y)}{C_{n}}=0
$$

Thus, in order that the rigid wall condition at $z=b, 0 \leq y \leq a$ is satisfied

$$
\psi(s, y, z)=\sum_{m=0}^{\infty} \frac{Y_{m}^{\prime}(d) Y_{m}(y) \cosh \left(\tau_{m}(s) z\right)}{C_{m} \tau_{m}(s) \sinh \left(\tau_{m}(s) b\right)}
$$

and, on imposing the condition of zero membrane displacement at $y=d, z=b$, the characteristic equation for the coupled modes is given by

$$
L(s)=\sum_{m=0}^{\infty} \frac{\left[Y_{m}^{\prime}(d)\right]^{2} \cosh \left(\tau_{m}(s) b\right)}{C_{m} \tau_{m}(s) \sinh \left(\tau_{m}(s) b\right)}=0
$$

\subsection{Orthotropic membrane}

The method used in Sec. 2-4.1 for obtaining the coupled eigenmodes works because the governing equation (2) and the boundary condition for an isotropic membrane (31) contain respectively the 3-D and 2-D Laplacian differential operator. This enables the problem to be recast in terms of the eigenvalues for the $y$ dependence. For an orthotropic membrane, however, this symmetry is spoilt and an alternative approach must be used. It transpires that, with minor modification to the membrane boundary condition, a standard Fourier method is sufficient. The modification is needed because the set of functions $\{\cos (m \pi z / b)\}_{m=0}^{\infty}$ are linearly independent and do not naturally accommodate the membrane edge conditions. Thus, the membrane condition must be replaced by

$$
\left\{\beta \frac{\partial^{2}}{\partial z^{2}}-\beta_{x} s^{2}+\mu^{2}\right\} \psi_{y}-\alpha[\psi]_{d^{-}}^{d^{+}}=\frac{2}{b} \sum_{n=0}^{\infty} \frac{(-1)^{n}}{\epsilon_{n}} \cos \left(\frac{n \pi z}{b}\right), 0 \leq z \leq b
$$

with $\beta_{x}=2 T_{x} /\left(T_{x}+T_{z}\right), \beta_{z}=2 T_{z} /\left(T_{x}+T_{z}\right), \mu=c_{a} / \sqrt{\left(T_{x}+T_{z}\right) /\left(2 \rho_{m}\right)}$ and $\alpha=$ $2 c_{a}^{2} \rho_{a} /\left[k\left(T_{x}+T_{z}\right)\right]$ where $T_{x}$ and $T_{z}$ are the tensions in the $x$ and $z$ directions respectively. (Note that, when $T_{x}=T_{z}$ these parameters reduce to their isotropic forms.) The divergent series on the right hand side of (39) is equal to an infinite series of delta functions[31], that is:

$$
\frac{1}{b} \sum_{n=0}^{\infty} \frac{(-1)^{n}}{\epsilon_{n}} \cos \left(\frac{n \pi z}{b}\right)=\sum_{m=-\infty}^{\infty} \delta(z-b-2 m b)
$$


in which the domain of $z$ is unrestricted. The concept of applying point forces in this manner in order to enforce specific conditions is standard practise, see for example reference [32].

The appropriate ansatz for this eigenfunction is

$$
\psi(s, y, z)=\sum_{n=0}^{\infty} F_{n}(s) Y_{n}(s, y) \cos (n \pi z / b)
$$

where $F_{n}(s)$ is as yet unknown. The piece-wise continuous (c.f. (26)) function $Y_{n}(s, y)$ satisfies $Y_{1}^{\prime}(s, 0)=Y_{2}^{\prime}(s, a)=0$ and continuity of normal velocity across the membrane. In this case: $Y_{1 n}(s, y)=\cosh \left(\nu_{n}(s) y\right)$ where, in view of $(2), \nu_{n}(s)=\left(s^{2}-1+n^{2} \pi^{2} / b^{2}\right)^{1 / 2}$ and

$$
Y_{2 n}(s, y)=\frac{\sinh \left(\nu_{n}(s) d\right)}{\sinh \left[\nu_{n}(s)(d-a)\right]} \cosh \left[\nu_{n}(s)(a-y)\right]
$$

On substituting (41) into (39), it is easily shown that

$$
\psi(s, y, z)=\frac{2}{b} \sum_{n=0}^{\infty} \frac{(-1)^{n+1} Y_{n}(s, y)}{\varepsilon_{n} K_{n}(s)} \cos (n \pi z / b)
$$

where $\varepsilon_{n}=2$ if $n=0$ and 1 otherwise, and

$$
\begin{gathered}
K_{n}(s)=\left(\beta_{x} s^{2}+\beta_{z} \frac{n^{2} \pi^{2}}{b^{2}}-\mu^{2}\right) \nu_{n}(s) \sinh \left(\nu_{n}(s) d\right) \\
-\frac{\alpha \sinh \left(\nu_{n}(s) a\right)}{\sinh \left[\nu_{n}(s)(a-d)\right]}=0
\end{gathered}
$$

Finally, in order to satisfy the zero displacement edge condition at $y=d, z=b$, the characteristic equation is

$$
L(s)=-\frac{2}{b} \sum_{n=0}^{\infty} \frac{\nu_{n}(s) \sinh \left(\nu_{n}(s) d\right)}{\varepsilon_{n} K_{n}(s)}=0 .
$$

Note that expressions (43) and (45) are independent of the roots of the underlying 2-D system but converge only for $\beta_{z}>0$.

Figures 9-10 show the phase speeds for the symmetric waves propagating in the drumlike silencer. For ease of comparison the parameters of Huang and Choy[11] are used. Thus, $\bar{a}=0.15 \mathrm{~m}, \bar{b}=0.05 \mathrm{~m}, \bar{d}=0.05 \mathrm{~m}$ and $\rho_{m} /\left(\rho_{a} \bar{d}\right)=1.4$. For such a small duct and for the frequency ranges displayed in figures 9-10, only the first of the uncoupled modes referred to in (30) propagates. This mode is, of course, the fundamental acoustic mode propagating with phase speed $344 \mathrm{~ms}^{-1}$ and is plotted as the dashed line in each graph. With regard to the coupled modes, there are clear differences between the three graphs in terms of the number of modes cut on at a specified frequency and the limiting values 

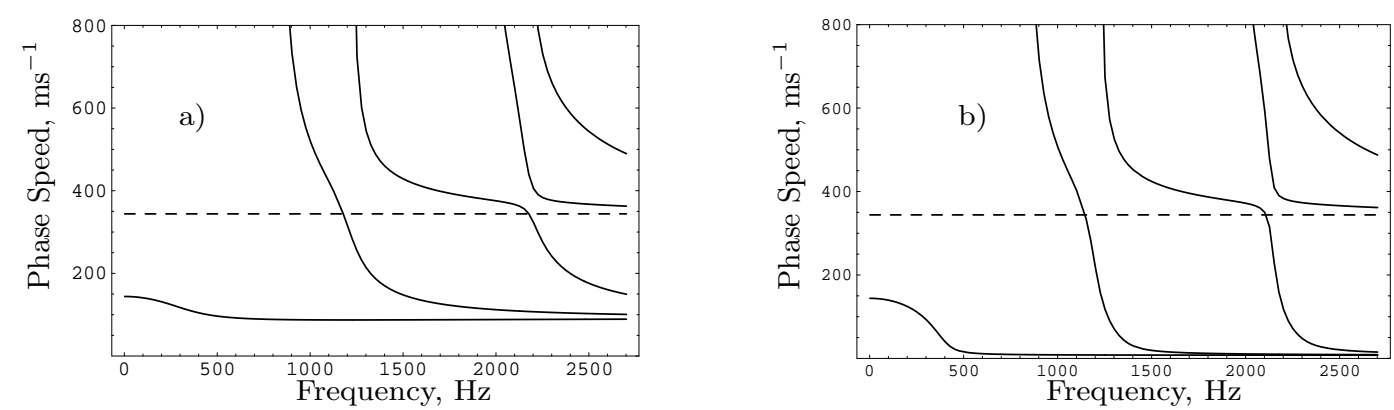

Figure 9: Drum-like silencer: phase speeds for a) $T_{z}=T_{x}=750$; b) $T_{x}=5, T_{z}=750$.

of the phase speeds as frequency increases. In figure 9(a) the membrane is isotropic with $T_{x}=T_{z}=750 \mathrm{~N}$ whilst in figure $9(\mathrm{~b})$ the membrane is orthotropic with $T_{x}=5 \mathrm{~N}$ and $T_{z}=750 \mathrm{~N}$. On comparing the two graphs, it is clear that each mode cuts on at approximately the same frequency and the major difference is the limiting value to which the phase speeds tend as frequency increases.

For figure 10 the membrane is again orthotropic with $T_{x}=750 \mathrm{~N}$ and $T_{z}=5 \mathrm{~N}$. For this configuration the phase speeds are significantly different from those of figure 9 . It is clear that the higher-order modes cut-on at much lower frequencies. Further, the phase speed for the fundamental coupled mode is almost identical to that observed in the 2-D drum-like silencer, that is the phase speed corresponding to first root of (33) which is depicted in figure 10 by triangular symbols. This is result consistent with the observations of Huang and Choy[11] who use the Galerkin procedure to study the drumlike silencer. They comment that with $T_{z}=0$ the 3 -D case behaves as for the $2-\mathrm{D}$ theory. It would appear, therefore, that for the duct size and the frequency range considered by Huang and Choy, the higher order modes evident in figure 10 have little effect on the sound field (such modes are not present in the 2-D case).

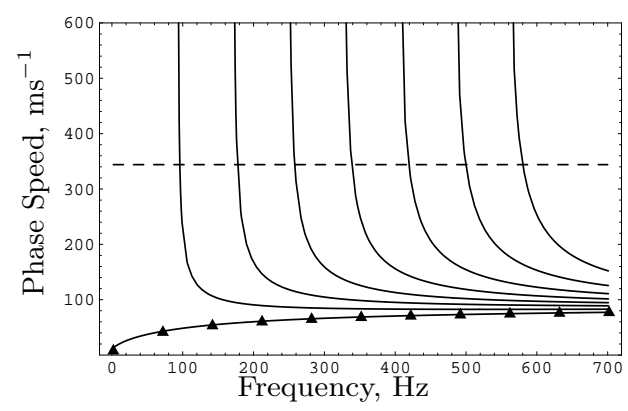

Figure 10: Drum-like silencer: phase speeds for $T_{x}=750, T_{z}=5$; triangles indicate that for the fundamental coupled mode of the corresponding 2-D case. 


\section{Discussion}

This article has presented analytic expressions for the characteristic equations and natural waveforms corresponding to acoustic propagation in a number of 3-D ducts of rectangular cross-section, each having one flexible wall. Such expressions enable the observation of various physical features (for example, the phase speeds) across a wide range of frequencies and duct dimensions. In this respect the approach offers an advantage over the Galerkin procedure [18, 11] and various other techniques [10].

In Sec. 2-4.1 the 3-D waveforms are expressed as a sum over the eigenmodes for the underlying 2-D system comprising the $x y$ cross-section. For the "drum-like" silencer with orthotropic membrane the 3-D eigenfunction is expressed as a sum over the eigenmodes for the underlying 2-D system comprising the $x z$ cross-section - that is, a Fourier cosine series. The latter has some advantages: in particular, no root-finding is necessary. For this reason it is worthwhile pointing out that all the 3-D waveforms considered herein can be represented in this manner [33]. For example, the symmetric eigenfunctions for the unlined 3-D duct with clamped corners considered in Sec. 2.2.1 may be expressed as

$$
\psi(s, y, z)=\frac{2}{b} \sum_{m=0}^{\infty} \frac{(-1)^{m} \cosh \left[\nu_{m}(s) y\right] \cos (m \pi z / b)}{\varepsilon_{m} K\left[\nu_{m}(s)\right]}
$$

where $\nu_{m}(s)=\left(s^{2}-1+m^{2} \pi^{2} / b^{2}\right)^{1 / 2}$ whilst the anti-symmetric eigenfunctions are given by

$$
\psi^{a}(s, y, z)=\frac{2}{b} \sum_{m=0}^{\infty} \frac{(-1)^{m} \cosh \left[\nu_{m}^{a}(s) y\right] \sin \left[\left(m+\frac{1}{2}\right) \pi z / b\right]}{K\left[\nu_{m}^{a}(s)\right]}
$$

where $\nu_{m}^{a}(s)=\left(s^{2}-1+\left(m+\frac{1}{2}\right)^{2} \pi^{2} / b^{2}\right)^{1 / 2}$. For both cases, $K\left[\nu_{m}^{(a)}(s)\right]=K(\gamma), \gamma=\nu_{m}^{(a)}(s)$ where $K(\gamma)$ is defined in (9). The corresponding characteristic equation is simply $\psi_{y}^{(a)}(s, a, b)=0$ where the subscript $y$ indicates differentiation with respect to this variable. Whilst these expressions converge well and offer the advantage that no root-finding is involved, the underlying eigensystem corresponding to the $x y$ cross-section cannot be entirely neglected as discussed below.

The eigenmodes presented in this article are essential to the development of hybrid analytic-numerical mode-matching (MM) methods for use with 3-D ducting systems comprising several different sections. A typical model geometry might, for example, comprise an unlined 3-D duct (of the type considered in Sec. 2.2) lying in the regions $|x|>\ell$; together with a silencing component (a lined duct of the type considered in Sec. 3) occupying $|x|<\ell$. In order to execute MM at the interfaces between consecutive duct sections (that is, at $x= \pm \ell$ ) appropriate generalised ORs are required. For the 
symmetric waveforms considered in Sec. 2.2.1 the appropriate result is generated using the OR for the eigenmodes of the underlying 2-D duct comprising the $x y$ cross-section. It is thus found $[25]$

$$
\begin{aligned}
\alpha \int_{0}^{b} \int_{0}^{a} \psi_{n}(y, z) \psi_{\ell}(y, z) d y d z & =D_{\ell} \delta_{\ell n} \\
-\int_{0}^{b}\left\{\psi_{n y y y}(a, z) \psi_{\ell y}(a, z)\right. & +\psi_{n y}(a, z) \psi_{\ell y y y}(a, z) \\
& \left.+2 \psi_{n y}(a, z) \psi_{\ell y}(a, z)\right\} d z
\end{aligned}
$$

where and $D_{n}=-L^{\prime}\left(s_{n}\right) /\left(2 s_{n}\right)$ (here $L(s)$ is given by (20) and the prime indicates differentiation with respect to the argument) and $\delta_{\ell n}$ is the Kronecker delta function. In view of the integral on the right hand side this is actually more of a partial OR rather than an OR. Nevertheless, its usefulness has been established by Lawrie [25] who considered a prototype model comprising a semi-infinite unlined 3-D duct with a prescribed pressure on the end-plate at $x=0,0 \leq y \leq a, 0 \leq z \leq b$. The analysis presented in that article indicates that the partial OR is likely to be sufficient to implement MM for situations in which the flexible surface of the duct is pin-jointed to the corresponding wall at the matching interface. For other edge conditions supplementary conditions are needed and these are presented in reference [25].

Of course, it is also necessary that the eigenfunction expansions generated by such MM schemes converge to the required physical solution. Fortunately, a number of important properties have been established [25] for the eigenmodes discussed in Sec. 2.2.1. In particular, they are linearly dependent:

$$
\sum_{n=0}^{\infty} \frac{\psi_{n y}(a, w) \psi_{n}(y, z)}{D_{n}}=\sum_{n=0}^{\infty} \frac{\psi_{n y y y}(a, w) \psi_{n}(y, z)}{D_{n}}=0
$$

and a modified Green's function (which takes into account the finite domains for $y$ and z) exists:

$$
\begin{aligned}
\sum_{n=0}^{\infty} \frac{\psi_{n}(y, z) \psi_{n}(v, w)}{D_{n}} & =\{\delta(v-y)+\delta(v+y)+\delta(v+y-2 a)\} \\
\times & \{\delta(w-z)+\delta(z+w)+\delta(w+z-2 b)\}
\end{aligned}
$$

where in both (49) and (50) $0 \leq v, y \leq a$ and $0 \leq w, z \leq b$. In (49), as mentioned above, the subscript $y$ indicates differentiation. These properties are sufficient to ensure the point-wise convergence of eigen-expansions of this class [25].

The derivations of (48)-(50) are crucially dependent on the properties of the 2-D eigenmodes $Y_{n}(y)$ discussed in Sec. 2.1. Since this is the case and much is known about 
this class of function[26], it is anticipated that results equivalent to (48)-(50) can be similarly constructed for the 3-D eigenmodes discussed in Sec. 3 and 4.1. It is thus concluded that sufficient tools are in place to enable the development of hybrid analyticnumerical MM schemes for a wide range of model problems involving the class of 3-D ducts considered in this article.

Finally, it is worth noting that theory presented herein also has application in the study of water waves. When the effects of surface tension are included, the free-surface boundary conditions are of high order (equivalent to a membrane). Thus, the waveforms for the 3-D wave-maker and related problems [34, 35] can be formulated in an analogous manner to those considered in Sec. 2.2-4.1. In this context the fluid-wall contact conditions replace the corner conditions relevant to this study. The motion of a fluid-solid contact line is, however, complicated and is not fully understood [36]. There is no single generally accepted form for the condition describing the contact line and a number of different conditions have been used in the literature. The article by Harter et al. [37] contains a good discussion of these, any of which can, in principle, be incorporated into the theory in a similar manner to the "clamped" or "pin-jointed" conditions of Sec. 2.2.

\section{References}

[1] K.S. Peat "The acoustical impedance at the junction of an extended inlet or outlet duct", J. Sound Vib. 150, 101-110 (1991).

[2] R. Kirby "A comparison between analytic and numerical methods for modelling automotive dissipative silencers with mean flow" J. Sound Vib. 325, 565-582 (2009).

[3] B. Nennig, E. Perrey-Debain, M. Ben Tahar "A mode matching method for modeling dissipative silencers lined with poroelastic materials and containing mean flow", J. Acoust. Soc. Am. 128, 3308-3320 (2010).

[4] A.D. Rawlins "Wave propagation in a bifurcated impedance-lined cylindrical waveguide", J. Eng. Math. 59, 419-435 (2007).

[5] J.B. Lawrie, I.D. Abrahams and C.M. Linton "Acoustic radiation from two opposed, semi-infinite, co-axial cylindrical waveguides, Part I: overlapping edges", Wave Motion 18(2), 121-142 (1993).

[6] A. McAlpine and M.C.M. Wright "Acoustic scattering by a spliced turbofan inlet duct liner at supersonic fan speeds", J. Sound Vib. 292, 911-934 (2006). 
[7] W. Bi, V. Pagneux, D. Lafarge and Aurégan Y. "An improved multimodal method for sound propagation in nonuniform lined ducts", J. Acoust. Soc. Am. 122, 280-290 (2007).

[8] B. Veitch and N. Peake "Acoustic propagation and scattering in the exhaust flow from coaxial cylinders", J. Fluid Mech. 613, 275-307 (2008).

[9] R.J. Astley, A. Cummings and N. Sormaz "A finite element scheme for acoustic propagation in flexible walled ducts with bulk-reacting liners, and comparison with experiment", J. Sound Vib. 150, 119-138 (1991).

[10] V. Martin, A. Cummings and C. Gronier "Discrimination of coupled structural/acoustical duct modes by active control: principles and experimental results", J. Sound Vib. 274, 583-603 (2004).

[11] L. Huang and Y.S. Choy "Vibroacoustics of three-dimensional drum silencer", J. Acoust. Soc. Am. 118, 2313-2320 (2005).

[12] J.B. Lawrie "Acoustic propagation in 3-D, rectangular ducts with flexible walls", INCE Conference Proceedings, 220(1), 1930-1938 (2010).

[13] B. Venkatesham, M. Tiwari and M.L. Munjal "Analytical prediction of the breakout noise from a rectangular cavity with one compliant wall ", J. Acoust. Soc. Am. 124, 2952-2962 (2008).

[14] B. Venkatesham, M. Tiwari and M.L. Munjal "Analytical prediction of the breakout noise from a reactive rectangular plenum with four flexible walls", J. Acoust. Soc. Am. 128, 1789-1799 (2010).

[15] J.B. Lawrie and I.D. Abrahams "A brief historical perspective of the Wiener-Hopf technique", J. Eng. Math., 59, 351-358 (2007).

[16] A.D. Rawlins 1997 "Two waveguide trifurcation problems", Math. Proc. Camb. Phil. Soc. 121(3), 555-573 (1997).

[17] I.D. Abrahams "Radiation and scattering of waves on an elastic half space; a noncommuatative matrix Wiener-Hopf problem", J. Mech. Phys. Solids 44(12), 2125-2154 (1996).

[18] L. Huang "Modal analysis of a drumlike silencer" J. Acoust. Soc. Am. 112, 20142025 (2002). 
[19] J.B. Lawrie and I.M.M. Guled "On tuning a reactive silencer by varying the position of an internal membrane", J. Acoust. Soc. Am. 120, 780-790 (2006).

[20] R. Kirby "Modeling sound propagation in acoustic waveguides using a hybrid numerical method", J. Acoust. Soc. Am. 124(4), 1930-1940 (2008).

[21] J.B. Lawrie and I.D. Abrahams "An orthogonality condition for a class of problem with high order boundary conditions; applications in sound/structure interaction", Q. J. Mech appl. Math. 52, 161-181 (1999).

[22] D.P. Warren, J.B. Lawrie and I.M. Mohamed "Acoustic scattering in wave-guides that are discontinuous in geometry and material property", Wave Motion 36, 119-142 (2002).

[23] E. Redon, A.-S. Bonnet-Ben Dhia, J.-F. Mercier and S. Poernomo Sari "Nonreflecting boundary conditions for acoustic propagation in ducts with acoustic treatment and mean flow" Int. J. Numer. Meth. Engng 86, 13601378 (2011).

[24] S.R. Manam, J. Bhattacharjee and T. Sahoo "Expansion formulae in wave structure interaction problems", Proc. R. Soc. Lond. A. 462, 263-287 (2006).

[25] J.B. Lawrie "Orthogonality relations for fluid-structural waves in a 3-D rectangular duct with flexible walls", Proc. R. Soc. A., 465, 2347-2367 (2009).

[26] J.B. Lawrie "On eigenfunction expansions associated with wave propagation along ducts with wave-bearing boundaries", IMA J. Appl. Math. 72, 376-394 (2007). (Equations (3.24) and (3.26) of reference [26] contain isolated sign errors - these do not affect the results presented therein.)

[27] M.E. Delany and E.N. Bazley "Acoustic properties of fibrous materials", Applied Acoustics 3, 105-116 (1970).

[28] R. Kirby and A. Cummings "Prediction of the bulk acoustic properties of fibrous materials at low frequencies", Applied Acoustics 56, 101-125 (1999).

[29] I.M.M. Guled "Acoustic transmission: waveguide with scattering or dissipative components", PhD Dissertation, Brunel University, U.K. (2003).

[30] Mahmood-Ul-Hassan, M.H. Meylan and M.A. Peters "Water-wave scattering by submerged elastic plates", Q. J. Mech. appl. Math. 62, 321-344 (2009).

[31] M.J. Lighthill "An introduction to Fourier analysis and generalised functions", Cambridge University Press. ISBN 0521091284 (1980) pp. 68. 
[32] J.B. Lawrie 'An infinite, elastic, cylindrical shell with a finite number of ring constraints", J. Sound Vib. 130, 189-206 (1989).

[33] J.B. Lawrie and R. Kirby "Mode-matching without root-finding: application to a dissipative silencer", J. Acoust. Soc. Am. 119, 2050-2061 (2006).

[34] S.C. Mohapatra, D. Karmakar and T. Sahoo, "On capillary gravity-wave motion in two-layer fluids" J. of Eng. Math. DOI 10.1007/s10665-011-9451-y (2011).

[35] M. Amaouche and B. Meziani "Oscillations of two superposed fluids in an open and flexible container", C.R. Mecaniques, 336, 329-335 (2008).

[36] J. Billingham "On a model for the motion of a contact line on a smooth solid surface", Euro. J. Appl. Math. 17, 347-382 (2006).

[37] R. Harter, M.J. Simon and I.D. Abrahams "The effect of surface tension on localized free-surface oscillations about surface-piercing bodies", Proc. R. Soc. A, 464, 30393054 (2008). 